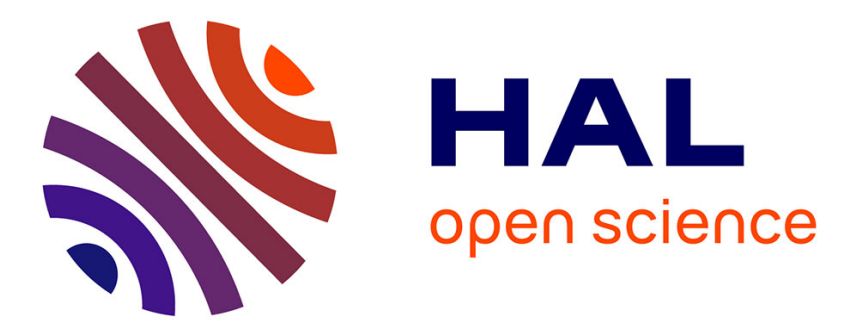

\title{
Root architecture and hydraulics converge for acclimation to changing water availability
}

Christophe Maurel, Philippe Nacry

\section{To cite this version:}

Christophe Maurel, Philippe Nacry. Root architecture and hydraulics converge for acclimation to changing water availability. Nature Plants, 2020, 6, pp.744-749. 10.1038/s41477-020-0684-5 . hal02885904

\section{HAL Id: hal-02885904 \\ https://hal.inrae.fr/hal-02885904}

Submitted on 16 Dec 2020

HAL is a multi-disciplinary open access archive for the deposit and dissemination of scientific research documents, whether they are published or not. The documents may come from teaching and research institutions in France or abroad, or from public or private research centers.
L'archive ouverte pluridisciplinaire HAL, est destinée au dépôt et à la diffusion de documents scientifiques de niveau recherche, publiés ou non, émanant des établissements d'enseignement et de recherche français ou étrangers, des laboratoires publics ou privés.

\section{(ㅇ)(1) $\$$}

Distributed under a Creative Commons Attribution - NonCommercial - NoDerivatives 44.0 


\section{Root architecture and hydraulics converge for acclimation to changing water \\ 2 availability}

3 Christophe Maurel* and Philippe Nacry

4 Biochimie et Physiologie Moléculaire des Plantes (BPMP)

5 Univ Montpellier, CNRS, INRAE, Institut Agro

62 2, Place Viala

7 F-34 060 Montpellier Cedex 2

8 France

*e-mail: christophe.maurel@cnrs.fr

Abstract. Because of intense transpiration and growth, the needs of plants for water can be immense. Yet, water in the soil is most often heterogeneous if not scarce due to more and more frequent and intense drought episodes. The converse context, flooding, is often associated with marked oxygen deficiency and can also challenge the plant water status. Under our feet roots achieve an incredible challenge to meet the water demand of the plant's aerial parts under such dramatically different environmental conditions. For this, they continuously explore the soil, building a highly complex, branched architecture. On shorter time scales, roots keep adjusting their water transport capacity (their so-called hydraulics), locally or globally. While the mechanisms that directly underlie root growth and development and tissue hydraulics are being uncovered, the signalling mechanisms that govern their local and systemic adjustments as a function of water availability remain largely unknown. A comprehensive understanding of root architecture and hydraulics as a whole (in other terms, root hydraulic architecture) is needed to apprehend the strategies used by plants to optimize water uptake and possibly improve crops for this crucial trait.

Word count: 3871

Water is certainly the most limiting environmental factor for agricultural production, whether in arid or temperate areas. Climate change exacerbates this threat by inducing increasingly frequent and intense drought episodes. While droughts can dramatically alter plant productivity and survival, water excess is not a lesser evil ${ }^{1}$. With notable exceptions such as rice and Rumex, complete 
submergence of the plant results in a full growth arrest ${ }^{1}$ and under waterlogging conditions several plant species exhibit a paradoxical wilting phenotype due to transpiration while root water uptake capacity has been reduced ${ }^{2}$. Thus, both water deficit and excess negatively impact agriculture and result in significant economical loses. For instance, a global analysis including drylands and nondrylands worldwide showed that crops as important as wheat and maize show a yield reduction by $21 \%$ and $39 \%$, respectively, at approximately $40 \%$ water reduction ${ }^{3}$. According to recent estimates, Europe loses up to 5 billion euros of agricultural harvests every year, due to river overflows. These losses may double by 2080 due to climate change ${ }^{4}$. Thus, understanding the overall modes of response of plant to water availability is of upmost agronomic importance ${ }^{1}$.

Several directions are currently followed, regarding water use. Foremost is irrigation, which has traditionally been used to counteract insufficient precipitation, although it is associated with significant economic and environmental costs. Soil drainage is also central to combat the detrimental effects of waterlogging ${ }^{5}$. Although novel water management and predictive practices ${ }^{5-7}$ have been explored to increase the efficiency of water usage ${ }^{8}$, developing drought- or flood-tolerant crop cultivars promises to be another main path to progress ${ }^{1,8,9}$. In particular, drought-tolerant crops are plants that are adapted to water-challenged climates that, most importantly, maintain a stable yield despite annual variations in precipitation. To reach this goal, breeders have focused on exploiting plant phenology as a means to protect the reproductive stage (the most drought-sensitive developmental stage in some crops). Breeding work has also been aimed at transpiration or leaf growth in order to prevent water loss, thereby achieving significant genetic progress in plant resistance to drought ${ }^{10}$. In contrast, most breeders have avoided the selection of root traits, although they fulfil key functions for the plant such as soil water uptake, which is absolutely essential during droughts and is challenged under waterlogging conditions. One reason is that roots vary with soil type and rainfall and require considerable investment for functional assays or growth monitoring in the field. Efforts to phenotype root systems in real soils, using X-ray computed tomography or shovelomics, or in adapted devices such as rhizotrons or transparent soils have led to significant technical progresses on how to capture the complexity and dynamics of root architecture and anatomy (see ${ }^{11}$ for a recent review). Yet, the functionality of root systems cannot be fully addressed using these phenotyping devices. In the present Perspective, we point to recent fundamental advances in root biology that open exciting avenues in several directions related to water uptake and ultimately crop improvement.

\section{Elucidating root hydraulic architecture}

Roots fulfil their anchoring and foraging functions through continuous growth and branching, thereby producing a highly complex and specialized network, the so called root system architecture 
(RSA) (Fig. 1a). Experimentally, RSA can be defined by length (depth) of the main root(s), the density and elongation of lower order roots, their gravitropic setpoint angle, and the possible presence of crown roots or adventitious roots. A large body of research has addressed the elementary mechanisms that determine each of these individual traits and emphasized the role of hormones, including auxin, abscisic acid (ABA), cytokinins and ethylene ${ }^{12-14}$. Other studies have addressed the adaptive significance of varying RSA. In a search for root ideotypes that could provide cereal crops with an adaptive advantage under drought, deep rooting has been proposed as a key trait, as it permits access to unexploited water resources when the soil surface desiccates ${ }^{15}$. Yet, this ideotype may not be optimal for quick recovery after drought in seasonal precipitation regimes ${ }^{16}$, or for acquisition of nutrients such as phosphate ${ }^{17}$. One initial approach to investigate the role of roots during drought consisted of searching for correspondences between Quantitative Trait Loci (QTLs) for specific root morphological traits and whole-plant performance under water deficit ${ }^{18,19}$. In grasses, for instance, suppression of crown roots was found to promote drought tolerance ${ }^{20,21}$. In addition, plant geneticists have recently succeeded in cloning such critical genes as rice DRO1, which determines root growth angle and thereby enhances rice performance under drought by favoring deep rooting ${ }^{22}$. A natural variation approach, but in Arabidopsis, led to identification of EXOCYST7OA3, an exocyst factor which also interferes with root growth orientation and root system depth by acting on expression of auxin efflux carriers in the root tip ${ }^{16}$. Although the two studies point to the central role of auxin in regulating RSA, they identify opposite and species-specific strategies for soil exploration under water deficit. More generally, much of the physiological and genetic components that shape the adaptive value of RSA under natural conditions are as yet unknown. To address these, elaborate time-lapse 3D imaging and mathematical modelling approaches are under development to capture the great complexity of RSA, and its intra- and interspecific variations ${ }^{23-25}$.

Water uptake is not simply a matter of root growth. It is also critically determined by the intrinsic water transport capacity of the roots, i.e. their hydraulics (Fig. 1b). Water is first transported radially from the soil to the stele, through concentric layers of root cells, loaded into xylem vessels, and then transported axially up to the shoots. Our biophysical and physiological understanding of these processes has made significant progress in the last two decades ${ }^{26,27}$. Molecular physiological studies have shown that water channel proteins named aquaporins facilitate water diffusion across cell membranes and therefore contribute to cell-to-cell transport ${ }^{26,28}$. Yet, many other components of root hydraulics are to be discovered. For instance, the crucial role of lignified and suberized barriers differentiated in the walls of exo- and endodermal cells has long remained elusive but can now be explored using genetic materials recently arising from sharp molecular dissection of these barriers ${ }^{29}$. Such studies have revealed however the intricate regulatory pathways connecting the cell 
wall (apoplastic) and cell-to-cell (aquaporin-dependent) water transport paths ${ }^{30}$. While root growth or leaf water relation traits have been the subject of extensive direct genetic analyses, it is only recently that these approaches have been extended to root hydraulics. For instance, QTL analysis of a biparental recombinant population of Arabidopsis led to the molecular cloning of Hydraulic Conductivity of Root 1 (HCR1) ${ }^{31}$. HCR1 encodes a raf-like MAP3K protein kinase which characterization has revealed unexpected hydraulic regulation under the combined effects of potassium and oxygen $\left(\mathrm{O}_{2}\right)$ availabilities (see below) (Fig. 2a,b). With respect to the strategy used for HCR1 mapping and cloning, Genome Wide Association Studies (GWAS) have recently provided a more direct approach for isolating genes controlling root hydraulic conductivity ${ }^{32}$. These studies uncovered XYLEM NAC DOMAIN 1 (XND1), a transcription factor that acts as a negative regulator of xylem differentiation and as a consequence of root hydraulics, thereby providing novel insights into the long-lasting debate on the possibly limiting role of xylem vessels in axial water transport (Fig. 1b). In relation with its effects on root water transport, XND1 negatively acts on drought stress tolerance ${ }^{32}$ (Fig. 2c). Overall, natural variation at HCR1 and XND1 points to the critical role of root hydraulics in plant adaptation to water excess or deficit in natural habitats. More broadly, these studies indicate how root hydraulics contributes to integrative response of plants to combined abiotic and biotic stresses (Fig. 2). Future development of such genetic approaches in crops will be crucial for dissecting and possibly improving root hydraulic performance under agricultural conditions.

Full comprehension of the ability of roots to capture soil water requires consideration of the root architecture and hydraulics as a whole. Mathematical modelling approaches have therefore attempted to integrate root local water transport properties within the RSA, thereby representing the so-called root hydraulic architecture ${ }^{33-35}$. Several recent works have aimed at a better match between root anatomy and architecture and water transport properties ${ }^{36-38}$. Nevertheless, there is still too little experimental data to support or challenge these models ${ }^{27,38-40}$. Consequently, although this is a crucial integrative trait for plant performance, root hydraulic architecture has largely been neglected by plant breeding efforts. In addition to RSA phenotyping, crude evaluation of root water uptake capacity in breeding materials, by direct measurements in pot or hydroponically grown plants ${ }^{41}$, or indirectly through transpiration in grafted species ${ }^{42}$, would certainly help estimate the potentialities of these materials. Another key issue is the interconnection between hydraulics and growth. While it is well supported in shoots, it is just emerging in roots. For instance, auxincontrolled aquaporins allow directing water flows in lateral primordia thereby reducing the mechanical resistance of overlaying cells and favoring the emergence of the newly formed lateral roots $^{43}$. This study establishes a unique physiological link between root hydraulics and branching.

\section{Root plasticity under varying water availability}


Root growth and hydraulics are both highly plastic, and are continuously adjusted in response to a large variety of soil signals with potentially antagonistic or synergistic effects. For instance, water and phosphorus deprivation can induce the formation of deep or shallow roots, respectively, while they both down-regulate aquaporins (root hydraulic conductivity) ${ }^{25,26}$. In addition, water and mineral resources are far from uniform and can dramatically vary between soil patches or strata. A key enigma in root research is therefore to resolve how plant roots, which lack a centralized information processing system, can integrate signals from a heterogeneous environment to design the optimal short- and long-term strategies for soil resource acquisition.

There is no need to say that, due to their great agronomical importance, these questions have been raising a strong interest. A large body of literature shows that water availability exerts multiple short- or long-term effects on root growth and hydraulics, with a sharp dose-dependency. For instance, moderate water deprivation enhances primary root growth and lateral root formation, whereas a strong water deficit exerts opposite inhibitory effects ${ }^{12,44,45}$. In addition, sensitivity of these responses can vary between the primary root and lateral roots of different orders, or between segments of a same axial root ${ }^{46}$. Root hydraulics is also dynamically regulated by water deficit, with species- or cultivar-specific profiles and, in most cases, an early inhibition of aquaporin function ${ }^{47,48}$. In maize, root aquaporins can also integrate diurnal changes in shoot water demand (transpiration), through a circadian regulation mechanism that shows a higher response amplitude under soil water deficit ${ }^{49}$. On a longer term, drought acts on root hydraulics by enhancing suberization of the endodermis, or interfering with xylem differentiation (Fig. 1a). For instance, water deficit triggers in the Arabidopsis root a non-cell-autonomous pathway involving endodermal ABA signaling. The hormone enhances the accumulation of microRNA165, which migrates into the stele to act on transcription factors determining xylem identity ${ }^{50}$.

Recently, there have been attempts to understand how these responses integrate with each other to determine whole root functionality. Analysis of both root architecture and hydraulics under exogenous-ABA treatments and in ABA-biosynthesis and signalling mutants has revealed striking parallels indicating that $A B A$, that accumulates under water deficit, acts as an integrator of root responses to the associated stress ${ }^{46}$. Yet, the picture is definitely more complex as ABA acts within a hormonal interaction network involving auxin, ethylene and cytokinins ${ }^{51,52}$. Future studies will also have to address the issue of local heterogeneity in water availability, which, besides overall water deficit, can occur in the absence of any water stress for the plant. Indeed, local variations can orient root growth (hydrotropism) ${ }^{53}$ or lateral root formation (hydropatterning) ${ }^{54,55}$ (Fig. 1a). Orman-Lipeza et al. ${ }^{56}$ recently described a related response, called xerobranching, which reflects the repression of lateral root formation when a root grows through a large air-filled soil macropore, thereby feeling a transient water deficit. In all cases, root tip growth and root branching are positioned towards 
regions of higher water availability (Fig. 1a). While much mechanistic details are being uncovered from laboratory experiments ${ }^{45}$, it will be fascinating to understand how these tropic responses mutually interact and operate during growth of roots in real, drying soils and how they allow optimizing soil foraging and water uptake.

Water excess reduces root hydraulic conductivity in most plant species, with early effects being mediated through proton-dependent gating of aquaporins due to metabolically-induced cell acidosis $^{2,57}$. On a longer term, water excess exerts profound effects on root anatomy and ultimately RSA. In some wetland species, hypoxia promotes suberin and lignin deposition in the outer layers of the roots (rhizodermis) to create a barrier for lateral diffusion of $\mathrm{O}_{2}$ from the root to the water logged soil ${ }^{58}$. More generally, and to facilitate root aeration, roots differentiate internal air spaces called aerenchyma, which can be either constitutive in species such as rice or inducible by hypoxia. While the role of ethylene and ROS in aerenchyma induction under hypoxic stress is now well assessed, a central role of auxin in constitutive aerenchyma formation was recently uncovered ${ }^{59}$. Interestingly, the auxin pathway involved shares commonalities with the AUX/IAA- and ARFdependent pathway responsible for lateral root formation. Yet, the two responses are somewhat distinct since auxin operates in two adjacent cell territories, e.g. cortex and pericycle. Concerning root growth, hypoxia can induce root slanting, to possibly escape from deeper layers in water logged soils ${ }^{60}$, due in part to a recently uncovered cytokinin-dependent mechanism that delays the gravitropic response of lateral roots ${ }^{61}$. More severe $\mathrm{O}_{2}$ deprivation can result in tip growth arrest if not death, and inhibition of lateral root formation ${ }^{1,58}$. To possibly alleviate defects of the primary root system, flooding also promotes the formation of adventitious roots at the shoot basis of floodtolerant species, a process that is primarily induced by tissue accumulation of ethylene ${ }^{1}$. While effects of energy depletion on transport activities in hypoxic roots have been largely identified ${ }^{62}$, the significance with respect to water uptake of hypoxia-induced root developmental reprogramming is as yet unclear. Thus, the notion of root hydraulic architecture in flooded plants will require as much attention as in droughted plants.

\section{Sensing water availability}

The molecular and cellular mechanisms underlying the early perception of water availability by roots and downstream signalling processes have become a crucial area in plant research. Interestingly, candidate genes involved in sensing water deficit are now emerging ${ }^{63,64}$ and associated signalling events involving ROS and calcium are being elucidated ${ }^{65,66}$. These studies, which deal with local effects of water deficit, typically relate to root responses to pronounced drought. Yet, the capacity of roots to acclimate to heterogeneous water resources or changing water demands of shoots, indicate 
that roots must continuously emit or sense systemic signals transferred within the root system or between roots and shoots ${ }^{67,68}$. A dehydration-induced peptide that relays root ABA signalling towards shoots was recently uncovered ${ }^{69}$ but other physical (e.g. hydraulic) or chemical (e.g. hormones, ions, ROS) signals are surely serving in root responses to heterogeneous or varying water availabilities .

Robbins and Dinneny ${ }^{70}$ have recently explored the initial steps of hydropatterning and used a thoughtful modelling approach to show that perception of water availability is necessarily linked to growth. In brief, they showed that growth allows to locally sustain a water potential gradient across the root radial plane whereas such gradient rapidly dissipates in a non-growing zone. These findings echo an independent experimental work showing that signal perception triggering the hydrotropic response of Arabidopsis roots occurs in the elongating cortex ${ }^{71}$. Thus, the general principles governing root responses to water availability are emerging and we now know where to look for 'water sensing' and early signalling mechanisms! A first significant step was recently taken by OrosaPuente et al. $^{72}$. These authors showed how the hydropatterning response relies on a chain of negative regulations which locally repress lateral root formation. In brief, local water deficit acts on SUMO protease function to enhance sumoylation of auxin response factor ARF7, which in turn accumulates and represses the downstream expression of root-inducing transcription factor LBD16. It is still disputed whether xerobranching is an extreme case of hydropatterning or would involve a fully distinct mechanism ${ }^{45,56}$. The authors showed that xerobranching can be mimicked by a transient $A B A$ accumulation and response due to local water deficit, which would somewhat antagonize constitutive auxin-dependent root branching. However, this model awaits validation using ABA signaling mutants ${ }^{45}$. Nevertheless, this hormonal regulation is different from previously described root responses to long-term exposure to $A B A$ or water deficit. In all cases, a deep interconnection between $A B A$ and auxin is emerging ${ }^{45}$.

The perception of water excess relies on a completely different mechanism involving a class (class VII) of transcription factors which are homologous to Ethylene Responsive Factors (ERF) and are specifically oxidized and degraded by the $\mathrm{O}_{2}$ and nitric oxide (NO) sensing branch of the PROTEOLYSIS 6 (PRT6) N-degron pathway ${ }^{1,73}$. Under flooding $\left(\mathrm{O}_{2}\right.$ deprivation) conditions, these socalled ERF-VII factors are stabilized to trigger a core anaerobic transcriptional response. Recent work indicate that this core mechanism is itself regulated by multiple hormonal and environmental cues. For instance, ethylene, which accumulates in the early phases of submergence and pre-acclimates plant to hypoxia stress, was shown to stabilize ERF-VII factors by increasing the expression of PHYTOGLOBIN1, which in turn scavenges NO ${ }^{74}$. The HCR1 protein kinase was also shown to stabilize one such factor (RAP2.12), specifically in the presence of $\mathrm{K}^{+}$, thereby potentiating this response, and promoting by as yet unknown mechanisms the inhibition of root hydraulic conductivity ${ }^{31}$. What 
might be the significance of this joint response pathway to $\mathrm{O}_{2}$ and $\mathrm{K}^{+}$availability? Whereas $\mathrm{O}_{2}$ deprivation directly reports on the intensity of flooding stress, $\mathrm{K}^{+}$availability could reflect the growth capacity of the plant depending on soil washing ${ }^{5}$. As a major intracellular cation, $\mathrm{K}^{+}$indeed contributes to turgor maintenance and thereby growth. In this context, HCR1 activation in the

242 presence of $\mathrm{K}^{+}$would promote the plant's metabolic acclimation to flooding stress, thereby providing 243 an enhanced growth capacity during the recovery phase (Fig. 2b). Depending on its intensity, flooding can result in soil water logging or full plant submergence that impact with different extent the water status and growth of plants ${ }^{1}$. It was proposed that natural alleles of HCR1 with distinct functionalities may have distinct adaptive values in these different scenarios.

ERF-VII transcription factors also play a role in the growth responses of Arabidopsis roots to hypoxia by repressing the slanting response of the primary root to hypoxia ${ }^{60}$ and promoting elongation of adventitious roots ${ }^{75}$. We also note that the capacity of plant roots to sense and accclimate to $\mathrm{O}_{2}$ availability goes beyond their responses to flooding stress. Hypoxic niches that naturally occur under aerobic conditions were recently shown to play significant roles in root development. Starting from the observation that hypoxic-responsive genes are induced during the late phases of lateral root formation, Shukla et al. ${ }^{76}$ showed how ERF-VII transcription factors bind to auxin-responsive transcriptional complexes to repress the expression of master regulators of lateral root formation, thereby contributing to the termination of this process.

\section{Conclusions}

Roots can be seen as a biological network integrating three main functions: growth, water transport, and water perception and signalling. The first two functions yield the root hydraulic architecture, an underexplored yet key trait. One main question is to understand how this architecture evolves in time and space by integrating local and systemic signals reporting on the availability of water. The present review indicates that the modes and mechanisms of response of roots to water deficit or excess are fundamentally distinct. While ABA emerges as a coordinator of root hydraulic architecture under drought ${ }^{46}$, we miss corresponding information on integrative hydraulic responses of roots to flooding stress. Also, a large focus has been made on aquaporin regulation under drought, whereas the significance of suberization and xylem differentiation with respect to root hydraulics remains disputed. More generally, future research will have to uncover the main principles and variety of strategies used by plant roots to optimize soil water uptake and maintain the plant water status under varying water availabilities. In these respects, mathematical modeling will be crucial to integrate functional and architectural components of whole root systems. In particular, a specific challenge will be to determine and possibly design root genotypes which are the best adapted to specific drought or flooding scenarios or culture practices such as deficit irrigation or partial root 
272 zone drying. More generally, a better integration of root functions and their relations to soil in crop 273 models would certainly aid crop improvement.

274 Recent progress in plant molecular genetics now makes it possible to directly address these 275 issues in the most relevant crops. Besides genome editing using CRISPR-Cas9 which now offers a 276 powerful approach to knock down genes of interest ${ }^{77}$, GWAS provide a potentially direct and rapid 277 approach to the identification of genes controlling a trait of interest. For instance, this approach has 278 been successfully used to isolate genes contributing to drought tolerance in crop plants with complex 279 genomes such as maize ${ }^{78,79}$ and it can now be used to target specific root traits. Cereals which 280 display an elaborate embryonic and postembryonic root developmental program also hold promises 281 for exciting discoveries in root functionalization. 
284 Fig. 1| The water uptake capacity of a root system is determined by both its architecture and 285 hydraulics.

286 a) The root system architecture is determined in part by soil water availability which can be highly 287 heterogeneous depending on soil layers. As shown in the inset, these local heterogeneities can 288 orientate root tip growth (hydrotropism) and lateral root formation (hydropatterning). b) Water 289 uptake can be decomposed in radial water transport from the soil to the root stele and axial 290 transport along the xylem vessels. Aquaporins, which contribute to transcellular water flows during 291 radial transport, determine to a large extent the root hydraulic conductivity. Suberized and lignified 292 endodermis or exodermis (not shown) can also impact radial transport.

Fig. 2| Quantitative genetics allowed the identification of novel genes involved in the regulation of root hydraulics under composite stress conditions.

a) Optimal growth and transpiration of a plant in a fully aerated $\left(\mathrm{O}_{2}\right)$, irrigated $\left(\mathrm{H}_{2} \mathrm{O}\right)$ and fertilized $\left(\mathrm{K}^{+}\right)$soil. The load of soil pathogenic bacteria (bacteria) is maintained at a minimum. The water uptake capacity of the root system is accounted for by its hydraulic conductivity $\left(L p_{r}\right)$. b) Flooding results in oxygen deficiency (hypoxia) of the root system. In the presence of nutriments $\left(\mathrm{K}^{+}\right)$, induction of the Hydraulic Conductivity of Root 1 (HCR1) pathway enhances the core anaerobic transcriptional response (anaerobic metabolism) and inhibits water uptake $\left(L p_{r}\right)$. These responses promote plant acclimation to submergence and a better growth during the recovery phase. c) The Xylem NAC Domain 1 (XND1) transcription factor negatively regulates xylem differentiation and as a consequence $L \mathrm{p}_{\mathrm{r}}$. Pathogenic bacteria can penetrate the root and move axially through its vasculature. They also enhance the expression of XND1. Reduced xylem differentiation is detrimental under water deficit (brown) and results in plant wilting. Yet, it restricts the proliferation of vascular pathogens and therefore diminishes the susceptibility of the plant to bacterial wilt. Thus, XND1 determines a trade-off between responses to abiotic and biotic stresses. 
3121 Voesenek, L. A. \& Bailey-Serres, J. Flood adaptive traits and processes: an overview. New $313 \quad$ Phytol 206, 57-73 (2015).

3142 Tan, X. et al. Plant water transport and aquaporins in oxygen-deprived environments. J Plant Physiol 227, 20-30 (2018).

3 Daryanto, S., Wang, L. \& Jacinthe, P. A. Global synthesis of drought effects on maize and wheat production. PLoS One 11, e0156362 (2016).

$4 \quad$ Hirabayashi, Y. et al. Global flood risk under climate change. Nature Climate Change 3, 816821 (2013).

5 Manik, S. M. N. et al. Soil and crop management practices to minimize the impact of waterlogging on crop productivity. Front Plant Sci 10, 140 (2019).

6 Du, T., Kang, S., Zhang, J. \& Davies, W. J. Deficit irrigation and sustainable water-resource strategies in agriculture for China's food security. J Exp Bot 66, 2253-2269 (2015).

$7 \quad$ Kirkegaard, J. A. et al. Improving water productivity in the Australian Grains industry-a nationally coordinated approach. Crop Pasture Sci. 65, 583-601 (2014).

8 Davies, W. J. \& Bennett, M. J. Achieving more crop per drop. Nature Plants 1, 15118 (2015).

9 Tester, M. \& Langridge, P. Breeding technologies to increase crop production in a changing world. Science 327, 818-822(2010).

10 Millet, E. J. et al. Genome-wide analysis of yield in Europe: Allelic effects vary with drought and heat scenarios. Plant Physiol 172, 749-764 (2016).

11 Atkinson, J. A., Pound, M. P., Bennett, M. J. \& Wells, D. M. Uncovering the hidden half of plants using new advances in root phenotyping. Curr Opin Biotechnol 55, 1-8 (2019).

12 Jung, J. K. \& McCouch, S. Getting to the roots of it: Genetic and hormonal control of root architecture. Front. Plant Sci. 4, 186 (2013).

13 Lavenus, J. et al. Lateral root development in Arabidopsis: fifty shades of auxin. Trends Plant Sci 18, 450-458 (2013).

14 Petricka, J. J., Winter, C. M. \& Benfey, P. N. Control of Arabidopsis root development. Annu Rev Plant Biol 63, 563-590 (2012).

15 Lynch, J. P. Steep, cheap and deep: an ideotype to optimize water and $\mathrm{N}$ acquisition by maize root systems. Ann Bot 112, 347-357 (2013).

16 Ogura, T. et al. Root system depth in Arabidopsis is shaped by EXOCYST70A3 via the dynamic modulation of auxin transport. Cell 178, 400-412 (2019).

17 Shahzad, Z. \& Amtmann, A. Food for thought: how nutrients regulate root system architecture. Curr. Opin. Plant. Biol. 39, 80-87 (2017).

18 Tuberosa, R. et al. Identification of QTLs for root characteristics in maize grown in hydroponics and analysis of their overlap with QTLs for grain yield in the field at two water regimes. Plant Mol. Biol. 48, 697-712 (2002).

19 Ruta, N., Liedgens, M., Fracheboud, Y., Stamp, P. \& Hund, A. QTLs for the elongation of axile and lateral roots of maize in response to low water potential. Theor Appl Genet 120, 621-631 (2010).

20 Gao, Y. \& Lynch, J. P. Reduced crown root number improves water acquisition under water deficit stress in maize (Zea mays L.). J Exp Bot 67, 4545-4557 (2016).

21 Sebastian, J. et al. Grasses suppress shoot-borne roots to conserve water during drought. Proc Natl Acad Sci USA 113, 8861-8866 (2016).

22 Uga, Y. et al. Control of root system architecture by DEEPER ROOTING 1 increases rice yield under drought conditions. Nat. Genet. 45, 1097-1102 (2013).

23 Jiang, N. et al. Three-dimensional time-lapse analysis reveals multiscale relationships in maize root systems with contrasting architectures. Plant Cell 31, 1708-1722 (2019).

24 Band, L. R. et al. Multiscale systems analysis of root growth and development: modeling beyond the network and cellular scales. Plant Cell 24, 3892-3906 (2012). 
25 Rellan-Alvarez, R., Lobet, G. \& Dinneny, J. R. Environmental control of root system biology. Annu Rev Plant Biol 67, 619-642 (2016).

26 Maurel, C. et al. Aquaporins in plants. Physiol. Rev. 95, 1321-1358 (2015).

27 Bramley, H., Turner, N. C., Turner, D. W. \& Tyerman, S. D. Roles of morphology, anatomy, and aquaporins in determining contrasting hydraulic behavior of roots. Plant Physiol. 150, 348364 (2009).

28 Hachez, C., Moshelion, M., Zelazny, E., Cavez, D. \& Chaumont, F. Localization and quantification of plasma membrane aquaporin expression in maize primary root: a clue to understanding their role as cellular plumbers. Plant Mol. Biol. 62, 305-323 (2006).

29 Barberon, M. et al. Adaptation of root function by nutrient-induced plasticity of endodermal differentiation. Cell 164, 447-459 (2016).

30 Wang, P. et al. Surveillance of cell wall diffusion barrier integrity modulates water and solute transport in plants. Sci. Rep. 9, 4227 (2019).

31 Shahzad, Z. et al. A potassium-dependent oxygen sensing pathway regulates plant root hydraulics. Cell 167, 87-98 (2016).

32 Tang, N. et al. Natural variation at XND1 impacts root hydraulics and trade-off for stress responses in Arabidopsis. Nat Commun. 9, 3884 (2018).

33 Doussan, C., Vercambre, G. \& Pages, L. Modelling of the hydraulic architecture of root systems: an integrated approach to water absorption - Distribution of axial and radial conductances in maize. Ann. Bot. 81, 225-232 (1998).

34 Doussan, C., Pages, L. \& Vercambre, G. Modelling of the hydraulic architecture of root systems: an integrated approach to water absorption - Model description. Ann. Bot. 81, 213223 (1998).

35 Lobet, G., Pages, L. \& Draye, X. A modeling approach to determine the importance of dynamic regulation of plant hydraulic conductivities on the water uptake dynamics in the soil-plant-atmosphere system. Ecol Model 290, 65-75 (2014).

36 Couvreur, V. et al. Going with the flow: multiscale insights into the composite nature of water transport in roots. Plant Physiol 178, 1689-1703 (2018).

37 Meunier, F., Couvreur, V., Draye, X., Vanderborght, J. \& Javaux, M. Towards quantitative root hydraulic phenotyping: novel mathematical functions to calculate plant-scale hydraulic parameters from root system functional and structural traits. J Math Biol 75, 1133-1170 (2017).

38 Zarebanadkouki, M., Kroener, E., Kaestner, A. \& Carminati, A. Visualization of root water uptake: quantification of deuterated water transport in roots using neutron radiography and numerical modeling. Plant Physiol 166, 487-499 (2014).

39 Pierret, A., Doussan, C. \& Pages, L. Spatio-temporal variations in axial conductance of primary and first order lateral roots of a maize crop as predicted by a model of the hydraulic architecture of root systems. Plant Soil 282, 117-126 (2006).

40 Draye, X., Kim, Y., Lobet, G. \& Javaux, M. Model-assisted integration of physiological and environmental constraints affecting the dynamic and spatial patterns of root water uptake from soils. J. Exp. Bot. 8, 2145-2155 (2010).

41 Matsuo, N., Ozawa, K. \& Mochizuki, T. Genotypic differences in root hydraulic conductance of rice (Oryza sativa L.) in response to water regimes. Plant Soil 316, 25-34 (2009).

42 Marguerit, E., Brendel, O., Lebon, E., Van Leeuwen, C. \& Ollat, N. Rootstock control of scion transpiration and its acclimation to water deficit are controlled by different genes. New Phytol 194, 416-429 (2012).

43 Péret, B. et al. Auxin regulates aquaporin function to facilitate lateral root emergence. Nature Cell Biol. 14, 991-998 (2012).

44 Deak, K. I. \& Malamy, J. Osmotic regulation of root system architecture. Plant J. 43, 17-28 (2005).

45 Dinneny, J. R. Developmental responses to water and salinity in root systems. Annu Rev Cell Dev Biol 35, 239-257 (2019). 
46 Rosales, M. A., Maurel, C. \& Nacry, P. Abscisic acid coordinates dose-dependent developmental and hydraulic responses of roots to water deficit. Plant Physiol 180, 21982211 (2019).

47 Vandeleur, R., Niemietz, C., Tilbrook, J. \& Tyerman, S. D. Role of aquaporins in root responses to irrigation. Plant Soil 274, 141-161 (2005).

48 Hachez, C. et al. Short-term control of maize cell and root water permeability through plasma membrane aquaporin isoforms. Plant Cell Environ 35, 185-198 (2012).

49 Caldeira, C. F., Jeanguenin, L., Chaumont, F. \& Tardieu, F. Circadian rhythms of hydraulic conductance and growth are enhanced by drought and improve plant performance. Nat Commun. 5, 5365 (2014).

50 Ramachandran, P., Wang, G., Augstein, F., de Vries, J. \& Carlsbecker, A. Continuous root xylem formation and vascular acclimation to water deficit involves endodermal ABA signalling via miR165. Development 145, dev.159202 (2018).

51 Rowe, J. H., Topping, J. F., Liu, J. \& Lindsey, K. Abscisic acid regulates root growth under osmotic stress conditions via an interacting hormonal network with cytokinin, ethylene and auxin. New Phytol 211, 225-239 (2016).

52 Li, X., Chen, L., Forde, B. G. \& Davies, W. J. The biphasic root growth response to abscisic acid in Arabidopsis involves interaction with ethylene and auxin signalling pathways. Front Plant Sci 8, 1493 (2017).

53 Moriwaki, T., Miyazawa, Y., Kobayashi, A. \& Takahashi, H. Molecular mechanisms of hydrotropism in seedling roots of Arabidopsis thaliana (Brassicaceae). Am. J. Bot. 100, 25-34 (2013).

54 Bao, Y. et al. Plant roots use a patterning mechanism to position lateral root branches toward available water. Proc. Natl. Acad. Sci. USA 111, 9319-9324 (2014).

55 von Wangenheim, D. et al. Early developmental plasticity of lateral roots in response to asymmetric water availability. Nat Plants 6, 73-77 (2020).

56 Orman-Ligeza, B. et al. The xerobranching response represses lateral root formation when roots are not in contact with water. Curr Biol 28, 3165-3173 e3165 (2018).

57 Tournaire-Roux, C. et al. Cytosolic pH regulates root water transport during anoxic stress through gating of aquaporins. Nature 425, 393-397 (2003).

58 Sauter, M. Root responses to flooding. Curr Opin Plant Biol 16, 282-286 (2013).

59 Yamauchi, T. et al. Fine control of aerenchyma and lateral root development through AUX/IAA- and ARF-dependent auxin signaling. Proc Natl Acad Sci USA 116, 20770-20775 (2019).

60 Eysholdt-Derzso, E. \& Sauter, M. Root bending is antagonistically affected by hypoxia and ERF-mediated transcription via auxin signaling. Plant Physiol 175, 412-423 (2017).

61 Waidmann, S. et al. Cytokinin functions as an asymmetric and anti-gravitropic signal in lateral roots. Nat Commun 10, 3540 (2019).

62 Shabala, S., Shabala, L., Barcelo, J. \& Poschenrieder, C. Membrane transporters mediating root signalling and adaptive responses to oxygen deprivation and soil flooding. Plant Cell Environ 37, 2216-2233 (2014).

63 Yuan, F. et al. OSCA1 mediates osmotic-stress-evoked $\mathrm{Ca}^{2+}$ increases vital for osmosensing in Arabidopsis. Nature 514, 367-371 (2014).

64 Hamilton, E. S. et al. Mechanosensitive channel MSL8 regulates osmotic forces during pollen hydration and germination. Science 350, 438-441 (2015).

65 Martiniere, A. et al. Osmotic stress activates two reactive oxygen species pathways with distinct effects on protein nanodomains and diffusion. Plant Physiol. 179, 1581-1593, (2019).

66 Shkolnik, D., Nuriel, R., Bonza, M. C., Costa, A. \& Fromm, H. MIZ1 regulates ECA1 to generate a slow, long-distance phloem-transmitted $\mathrm{Ca}^{2+}$ signal essential for root water tracking in Arabidopsis. Proc Natl Acad Sci USA 115, 8031-8036 (2018). 
Puertolas, J., Conesa, M. R., Ballester, C. \& Dodd, I. C. Local root abscisic acid (ABA) accumulation depends on the spatial distribution of soil moisture in potato: implications for ABA signalling under heterogeneous soil drying. J Exp Bot 66, 2325-2334 (2015).

68 McLean, E. H., Ludwig, M. \& Grierson, P. F. Root hydraulic conductance and aquaporin abundance respond rapidly to partial root-zone drying events in a riparian Melaleuca species. New Phytol 192, 664-675 (2011).

69 Takahashi, F. et al. A small peptide modulates stomatal control via abscisic acid in longdistance signalling. Nature 556, 235-238 (2018).

70 Robbins, N. E., 2nd \& Dinneny, J. R. Growth is required for perception of water availability to pattern root branches in plants. Proc Natl Acad Sci USA 115, E822-E831 (2018).

71 Dietrich, D. et al. Root hydrotropism is controlled via a cortex-specific growth mechanism. Nature Plants 3, 17057 (2017).

72 Orosa-Puente, B. et al. Root branching toward water involves posttranslational modification of transcription factor ARF7. Science 362, 1407-1410 (2018).

73 Holdsworth, M. J., Vicente, J., Sharma, G., Abbas, M. \& Zubrycka, A. The plant N-degron pathways of ubiquitin-mediated proteolysis. J Integr Plant Biol 62, 70-89 (2019).

74 Hartman, S. et al. Ethylene-mediated nitric oxide depletion pre-adapts plants to hypoxia stress. Nat Commun 10, 4020 (2019).

75 Eysholdt-Derzso, E. \& Sauter, M. Hypoxia and the group VII ethylene response transcription factor HRE2 promote adventitious root elongation in Arabidopsis. Plant Biol (Stuttg) 21 Suppl 1, 103-108 (2019).

76 Shukla, V. et al. Endogenous hypoxia in lateral root primordia controls root architecture by antagonizing auxin signaling in Arabidopsis. Mol Plant 12, 538-551 (2019).

77 Char, S. N. et al. An Agrobacterium-delivered CRISPR/Cas9 system for high-frequency targeted mutagenesis in maize. Plant Biotechnol J 15, 257-268 (2017).

78 Wang, X. et al. Genetic variation in ZmVPP1 contributes to drought tolerance in maize seedlings. Nat Genet 48, 1233-1241 (2016).

79 Mao, H. et al. A transposable element in a NAC gene is associated with drought tolerance in maize seedlings. Nat Commun 6, 8326 (2015).

\section{Acknowledgements}

This work was supported in part by the Agence Nationale de la Recherche (ANR-11-BSV6-018) and the European Research Council (ERC-2017-ADG- 788553).

\section{Contributions}

C.M. wrote this article which was discussed with and corrected by P.N.

\section{Corresponding author}

Correspondence to Christophe Maurel

\section{Competing interests}

The authors declare no competing interests 
a

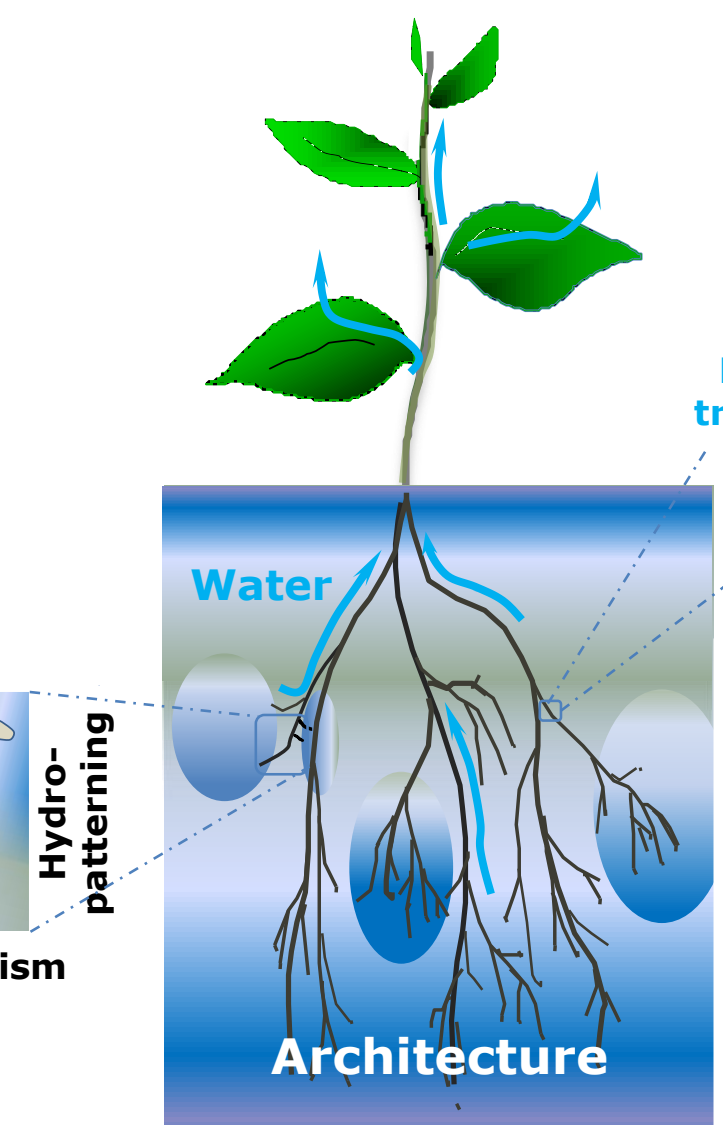

\section{Hydrotropism}

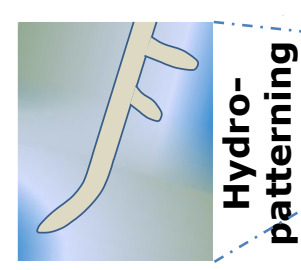

b

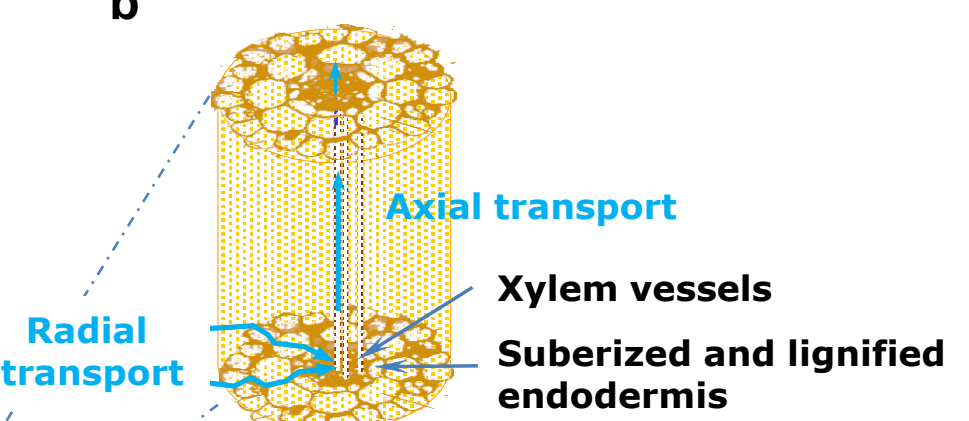
endodermis
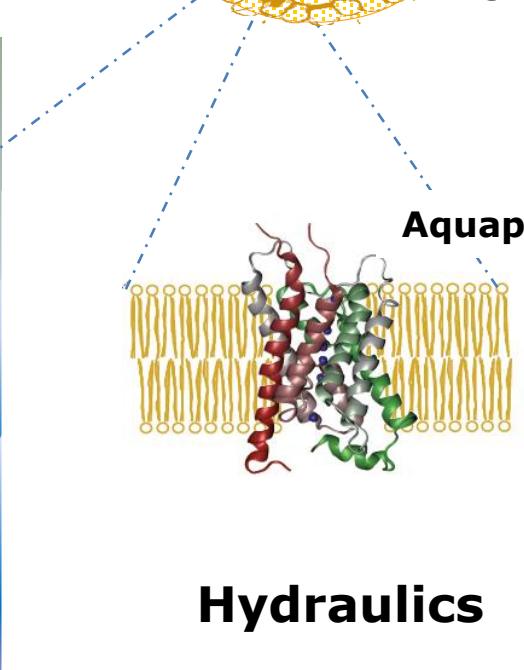

Figure 1 
a

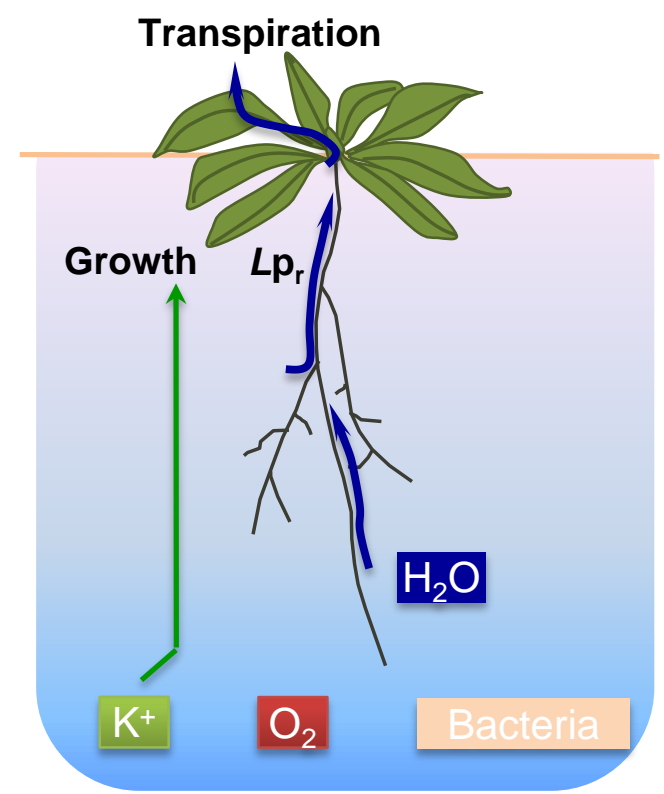

b

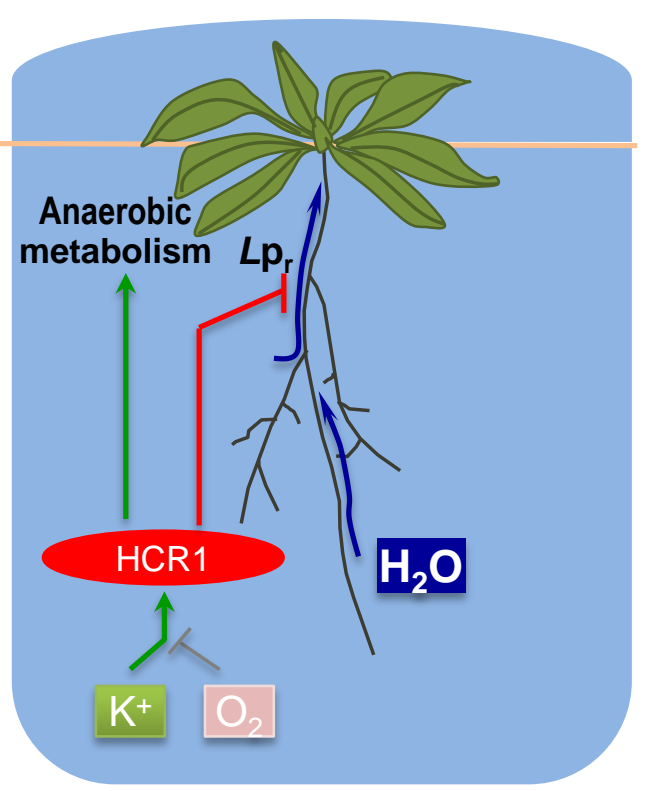

C

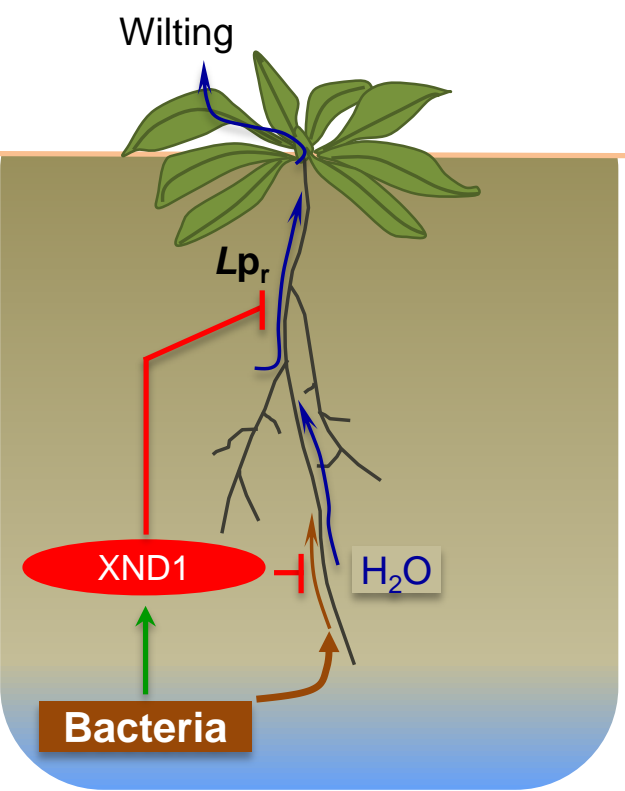

